\title{
Inhibition of Forkhead box protein M1 by thiostrepton increases chemosensitivity to doxorubicin in T-cell acute lymphoblastic leukemia
}

\author{
JIAN-YONG WANG ${ }^{1 *}$, XIU-HONG JIA ${ }^{1 *}$, HAI-YAN XING ${ }^{2}$, YOU-JIE LI $^{3}$, \\ WEN-WEN FAN ${ }^{4}$, NA LI $^{1}$ and SHU-YANG XIE ${ }^{3}$ \\ ${ }^{1}$ Department of Pediatrics, The Affiliated Hospital of Binzhou Medical University, Binzhou, Shandong 256603; \\ ${ }^{2}$ Department of Respiration, Binzhou People's Hospital, Binzhou, Shandong 256603; \\ ${ }^{3}$ Department of Biochemistry and Molecular Biology, Key Laboratory of Tumour Molecular Biology, \\ Binzhou Medical University, Yantai, Shandong 264003; ${ }^{4}$ Department of Pediatrics, \\ Women and Children Hospital of Qingdao, Qingdao, Shandong 266011, P.R. China
}

Received June 13, 2014; Accepted February 27, 2015

DOI: $10.3892 / \mathrm{mmr} .2015 .3469$

\begin{abstract}
T-cell acute lymphoblastic leukemia (T-ALL) is an aggressive type of blood malignancy, deriving from T-cell progenitors in the thymus, and comprises $10-15 \%$ of pediatric and $25 \%$ of adult primary ALL cases. Despite advances, $20 \%$ of pediatric and the majority of adult patients with T-ALL succumb to mortality from resistant or relapsed disease, and the survival rate for patients with resistant or relapsed T-ALL remains poor. Alterations in the expression of Forkhead box protein M1 (FoxM1) have been detected in several types of cancer, and the inhibition of FoxM1 has been investigated as therapeutic strategy in cancer. The present study investigated the effects of the inhibition of FoxM1 by thiostrepton in human T-ALL Jurkat cells. The cells were treated with different concentrations of thiostrepton, either alone or in combination with doxorubicin. Cell viability was measured using CCK-8 assays and the cell cycle distribution, apoptosis and cell-associated mean fluorescence intensity of intracellular doxorubicin were assessed using flow cytometric analysis. The mRNA and protein expression levels were detected by reverse transcription-quantitative polymerase chain reaction and western blot analyses. The inhibition of FoxM1 by thiostrepton significantly decreased the proliferation of the Jurkat cells proliferation in a time- and dose-dependent manner. Cell arrest at the G2/M phase, and apoptosis was significantly increased
\end{abstract}

Correspondence to: Professor Xiu-Hong Jia, Department of Pediatrics, The Affiliated Hospital of Binzhou Medical University, 661 Yellow River 2nd Road, Binzhou, Shandong 256603, P.R. China E-mail: jiaxiuhong001@163.com

${ }^{*}$ Contributed equally

Key words: thiostrepton, Forkhead box protein M1, T-cell acute lymphoblastic leukemia, doxorubicin in the thiostrepton-treated Jurkat cells. Thiostrepton reduced the half maximal inhibitory concentration of doxorubicin in the Jurkat cells, and significantly enhanced the cytotoxicity of doxorubicin within the Jurkat cells by enhancing doxorubicin-induced apoptosis and increasing the accumulation of intracellular doxorubicin. Furthermore, the inhibition of FoxM1 by thiostrepton enhanced doxorubicin-induced apoptosis, possibly through a caspase-3-dependent pathway, and increased the accumuation of intracellular doxorubicin, possibly through downregulating the expression of glutathione S-transferase pi. Collectively, the results of the present study suggested that targeting FoxM1 with thiostrepton resulted in potent antileukemia activity and chemosensitizing effects in human T-ALL Jurkat cells.

\section{Introduction}

Acute lymphoblastic leukemia (ALL) occurs most frequently in children, with incidence peaks between the ages of 2 and 5 years, and is one of the most common childhood malignancies worldwide $(1,2)$. T-cell ALL (T-ALL) is an aggressive type of blood malignancy, deriving from T-cell progenitors in the thymus, and comprises $10-15 \%$ of pediatric and $25 \%$ of adult primary ALL cases (3). The age of the patient at diagnosis, leukocyte count, ethnicity, gender and immunophenotype are clinical prognostic parameters, which are utilized to classify the patients into different risk groups (1). T-ALL is classified as a high-risk group in ALL (2). With the currently used intensive multi-agent chemotherapeutic procedures, the 5-year event-free survival (EFS) of children with T-ALL is $70-75 \%$, whereas the EFS is $30-40 \%$ for adults $<60$ years of age, and $10 \%$ in those $>60$ years old (4). However, these types of therapy are highly toxic, and cases of relapse often develop resistance to chemotherapy, with a poor prognosis (2). Targeting leukemia-specific molecular abnormalities may further improve outcomes and reduce the adverse side effects of therapy.

Forkhead box protein M1 (FoxM1), characterized by a 100 amino acid winged-helix DNA binding domain, is a newly 
unified family member of the Forkhead transcription factors (5). The protein expression of FoxM1 is usually restricted to proliferating cells and is absent in quiescent or terminally differentiated cells, however, elevated expression levels of FoxM1 have been detected in a wide range of different types of human tumor and has been implicated in cellular transformation, tumor initiation and progression (6). By regulating the G1/S and G2/M transitions and $\mathrm{M}$ phase progression, antagonizing cellular senescence, stimulating stem cell-like characteristics (including self-renewal), promoting multiple steps of cancer progression by inducing mitogenic and survival signals, and promoting tumor invasion, migration, and angiogenesis, FoxM1 is important in tumorigenesis and cancer progression (6). Accumulating evidence has demonstrated that increased expression levels of FoxM1 are associated with a poor prognosis in various types of cancer (7-11), including acute myeloid leukemia (AML) (12). In addition, FoxM1 deregulation has also been associated with the development of cancer drug resistance (13). A previous study confirmed that suppression of FoxM1 enhanced the chemosensitivity of various types of cancer cell to the DNA-damaging reagent, doxorubicin (14). Although a number of investigations have examined the role of FoxM1 in AML (12,15-17), the role of FoxM1 in ALL, particularly pediatric ALL, remains to be elucidated.

Thiostrepton, a natural product originally isolated from Streptomyces azureus, has received significant attention due to its potent anticancer activity as a FoxM1 inhibitor $(18,19)$. Thiostrepton can downregulate the mRNA and protein expression levels of FoxM1 (20). The mechanism by which thiostrepton affects FoxM1 has been confirmed. Thiostrepton hinders the proteasomal degradation of a negative regulator of FoxM1 (NRFM), which either directly or indirectly inhibits the activity of FoxM1 as a transcription factor (21). Notably, thiostrepton appears to exert minimal toxicity against non-malignant cells (22). Thiostrepton has exhibited anticancer activity in rodent xenograft models, without observable toxicity $(23,24)$. Collectively, these data suggest that thiostrepton is a suitable treatment for T-ALL, particularly in children. In the present study, the antileukemia effects of thiostrepton were examined in human T-ALL Jurkat cells. Crucially, the ability of thiostrepton to sensitize Jurkat cells to doxorubicin, which is commonly used for the treatment of ALL, was assessed.

\section{Materials and methods}

Chemicals and reagents. Thiostrepton (off-white powder, $1 \mathrm{~g}$ ), purchased from Merck Millipore (Darmstadt, Germany), was freshly dissolved in dimethyl sulfoxide (DMSO; Sigma-Aldrich, St. Louis, MO, USA) to produce a $20 \mathrm{mmol} / 1$ stock solution. Doxorubicin (Haizheng Medicine Co. Ltd., Zhengjiang, China) was dissolved in a stock concentration of $10 \mathrm{mmol} / \mathrm{l}$ with $\mathrm{ddH}_{2} \mathrm{O}$ and divided into five aliquots. Rabbit monoclonal antibody against FoxM1 (cat. no. 5436) was obtained from Cell Signaling Technology, Inc. (Danvers, MA, USA). Rabbit polyclonal antibodies against glutathione S-transferase pi (GSTpi; cat. no. PB0144), survivin (cat. no. BA4055-2), caspase-3 (cat. no. BA2885-2) and $\beta$-actin (cat. no. BA2305) were obtained from Boster Biological Technology, Ltd. (Wuhan, China).
Cell lines and cell culture. The human T-ALL Jurkat cells (Key Laboratory of Tumour Molecular Biology of Binzhou Medical University (Binzhou, China) were cultured in RPMI-1640 medium (HyClone Laboratories, Inc., Logan, UT, USA), supplemented with $10 \%$ heat-inactivated fetal bovine serum (FBS) (GE Healthcare Bio-Sciences, Logan, UT, USA) at $37^{\circ} \mathrm{C}$ in $5 \% \mathrm{CO}_{2}$.

Cell viability assay. Cell viability was assessed using a Cell Counting Kit-8 (CCK-8; Dojindo Molecular Technologies, Inc., Shanghai, China), according to the manufacturer's instructions. The Jurkat cells were seeded into 96-well flat plates $\left(1.5 \times 10^{4}\right.$ cells/well $)$ and then treated with or without various concentrations of thiostrepton $(0,1,2$ or $3 \mu \mathrm{m} / \mathrm{l})$ for 24,48 or $72 \mathrm{~h}$ at $37^{\circ} \mathrm{C}$. The cells in the control group were treated with the equivalent quantity of dimethyl sulfoxide, at a final concentration of $0.02 \%$. The CCK-8 kit was then used, according to the manufacturer's instructions. The optical density (OD) was measured at $450 \mathrm{~nm}$ using a fluorescence spectrofluorometer (F-7000; Hitachi High-Technologies Corporation, Tokyo, Japan), and the reference OD was subtracted. RPMI-1640 containing 10\% FBS with $0.02 \%$ DMSO served as the reference group. Cell viability was then calculated as follows: Cell viability = $\left(\mathrm{OD}_{\text {sample }}-\mathrm{OD}_{\text {reference }}\right) /\left(\mathrm{OD}_{\text {control }}-\mathrm{OD}_{\text {reference }}\right) \times 100 \%$. The half maximal inhibitory concentration of cells $\left(\mathrm{IC}_{50}\right)$ was obtained using a probit regression analysis method (25). Each experiment was performed in triplicate and repeated three times.

Cell cycle analysis. The Jurkat cells were seeded into 6-well plates in RPMI-1640, containing $10 \%$ fetal bovine serum (FBS), with or without the agents, for $24 \mathrm{~h}$ at $37^{\circ} \mathrm{C}$. The cells were harvested and fixed with $500 \mu 170 \%$ cold $\left(-20^{\circ} \mathrm{C}\right)$ ethanol (Shenzhen Kingstone Experimental Equipment Co., Ltd., Shenzhen, China) at $4^{\circ} \mathrm{C}$ overnight, and then incubated with RNase A (KeyGen Biotech Co., Ltd., Nanjing, China) for $30 \mathrm{~min}$ at $37^{\circ} \mathrm{C}$, followed by incubation with $100 \mu \mathrm{g} / \mathrm{ml}$ propidium iodide (PI; KeyGen Biotech Co., Ltd.) at room temperature for $30 \mathrm{~min}$. The cell cycle distribution was detected using a flow cytometer (FACS FC500; Beckman Coulter, Brea, CA, USA).

Detection of apoptosis. The Jurkat cells $\left(2.5 \times 10^{5}\right.$ cells $\left./ \mathrm{ml}\right)$ were seeded into 6-well plates in RPMI-1640 containing 10\% FBS, with or without the agents, for $24 \mathrm{~h}$. Tells were collected and washed twice with phosphate-buffered saline (PBS), suspended in $500 \mu \mathrm{l}$ binding buffer (KeyGen Biotech Co.,Ltd.), $5 \mu \mathrm{l}$ annexin V-fluorescein isothiocyanate and $5 \mu \mathrm{l}$ PI (KeyGen Biotech Co., Ltd.) for $15 \mathrm{~min}$ in the dark at room temperature. The apoptotic cells were analyzed using a flow cytometer.

Reverse transcription-quantitative polymerase chain reaction $(R T-q P C R)$. Total RNA was isolated from the cells using TRIzol reagent (Invitrogen Life Technologies, Carlsbad, CA, USA). RT into complementary DNA (cDNA) was performed using a PrimeScript ${ }^{\mathrm{TM}}$ RT reagent kit with gDNA eraser (Takara Bio, Inc., Otsu, Japan). qPCR was performed using a SYBR ${ }^{\circledR}$ Premix Ex Taq ${ }^{\mathrm{TM}}$ kit (Takara Bio, Inc.) on an ABI PRISM 7500 real-time PCR system (Applied Biosystems, Foster City, CA, USA). The cycling parameters were as follows: $95^{\circ} \mathrm{C}$ for $30 \mathrm{sec}$, 
Table I. Primers used in reverse transcription-quantitative polymerase chain reaction.

\begin{tabular}{llc}
\hline Gene & \multicolumn{1}{c}{ Primer sequence } & Product length (bp) \\
\hline FoxM1 & F: 5'-AGGAAGTGGCAGAGTCCAAC-3' & 128 \\
& R: 5'-TGCTGTGATGATGCTGTGAA-3' & 127 \\
GSTpi & F: 5'-CACTCAAAGCCTCCTGCCTA-3' & 131 \\
& R: 5'-TGCTGGTCCTTCCCATAGAG-3' \\
& F: 5'-TGGTATCGTGGAAGGACTCA-3' & R: 5'-CAGTAGAGGCAGGGATGATG-3' \\
\hline
\end{tabular}

FoxM1, forkhead box protein M1; GSTpi, glutathione S-transferase pi; F, forward; R, reverse.

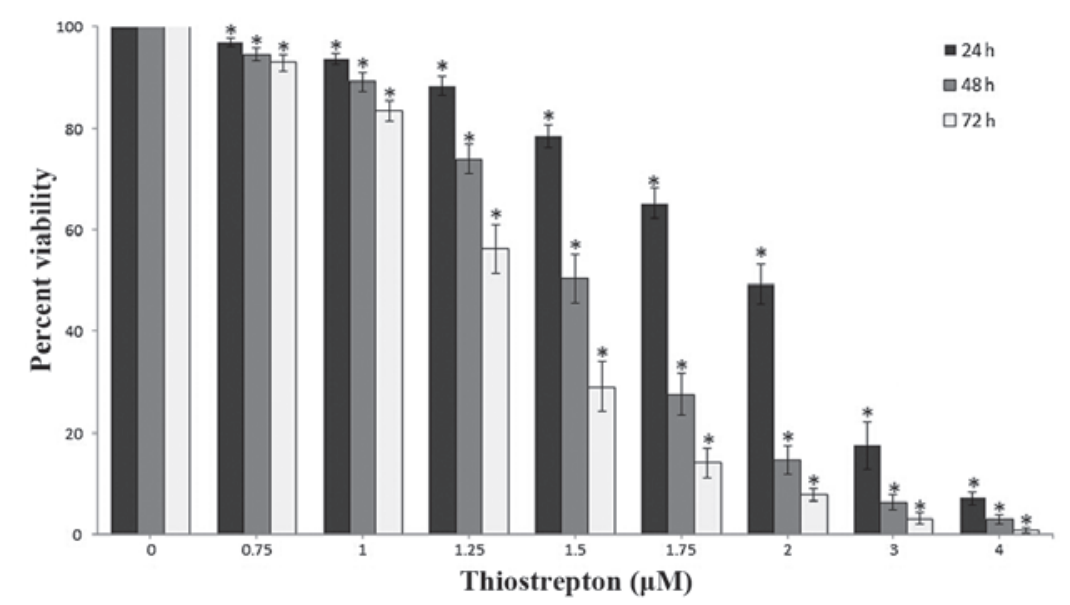

Figure 1. Effect of thiostrepton on the viability of Jurkat cells. Jurkat cells were treated with various concentrations of thiostrepton $(0-4 \mu \mathrm{M})$ for 24,48 and $72 \mathrm{~h}$. Cell viability was assessed using a Cell Counting kit- 8 assay. Data are presented as the percentages relative to the untreated control, as the means \pm standard deviation. " $\mathrm{P}<0.05$ vs. untreated control.

then 40 cycles of $95^{\circ} \mathrm{C}$ for $5 \mathrm{sec}, 60^{\circ} \mathrm{C}$ for $34 \mathrm{sec}$. The isolation of RNA, RT and qPCR were performed, according to the manufacturer's instructions. The primers used (Table I) were designed using Primer 5 version 5.6.0 software and synthesized by Sangon Biotech Co, Ltd. (Shanghai, China). GAPDH was used as an internal control. qPCR for each gene of each cDNA sample was assayed in triplicate. The results were calculated using the $2^{-\Delta \Delta \mathrm{Ct}}$ method. The following equations were used: $\Delta \mathrm{Ct}=\mathrm{Ct}_{\text {(target gene) }}-\mathrm{Ct}_{\text {(actin) }} ; \Delta \Delta \mathrm{Ct}=\Delta \mathrm{Ct}_{\text {(thiostrepton-treated }}$ cells) $-\Delta \mathrm{Ct}_{\text {(untreated control) }}$.

Western blot analysis. The ells were lysed in $100 \mu \mathrm{l}$ radioimmunoprecipitation assay buffer (Beyotime Biotechnology, Shanghai, China)., supplemented with $1 \mu 1$ phenylmethylsulfonyl fluoride (Beyotime Biotechnology), and the protein concentration of the lysate was determined using a Bicinchoninic Acid Protein Assay kit (Beyotime Biotechnology). The proteins (50 $\mu \mathrm{g} /$ lane) were separated by 8-12\% SDS-PAGE (Beyotime Biotechnology) and transferred to polyvinylidine difluoride membranes (EMD Millipore, Bedford, MA, USA). The membranes were blocked with 5\% skimmed milk for $2 \mathrm{~h}$, and then incubated overnight at $4^{\circ} \mathrm{C}$ with specific antibodies. The primary antibodies used were rabbit monoclonal antibody against FoxM1 (1:1,000), and rabbit polyclonal antibodies against GSTpi (1:200), survivin (1:200),
Table II. Interaction between doxorubicin and thiostrepton in combined treatment with different doses of doxorubicin.

\begin{tabular}{lr}
\hline Doxorubicin $(\mu \mathrm{M})^{\mathrm{a}}$ & Q-value \\
\hline 0.025 & 0.86 \\
0.05 & 1.17 \\
0.1 & 1.32 \\
0.2 & 1.61 \\
0.3 & 1.43 \\
0.4 & 1.25 \\
0.8 & 1.07
\end{tabular}

$\mathrm{Q}>1.15$ indicates to a synergistic interaction; ${ }^{\mathrm{a}} \mathrm{At}$ each dose of doxorubicin, $1 \mu \mathrm{M}$ thiostrepton was used.

caspase-3 (1:200) and $\beta$-actin (1:1,000). The following day, the membrane was incubated in horesradish peroxidase-labeled goat anti-rabbit immunoglobulin G (1:5,000; cat. no. ZB-5301; Beijing ZhongShan-Golden Bridge Technology Co., Ltd., Beijing, China) for $1 \mathrm{~h}$ at room temperature. Finally, images were captured using a FluorChem FC2 gel imaging system (Alpha Innotech, San Leandro, CA, USA). 
A

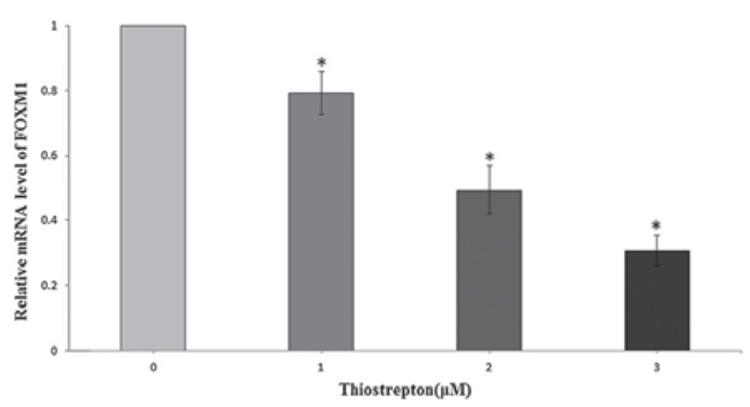

C

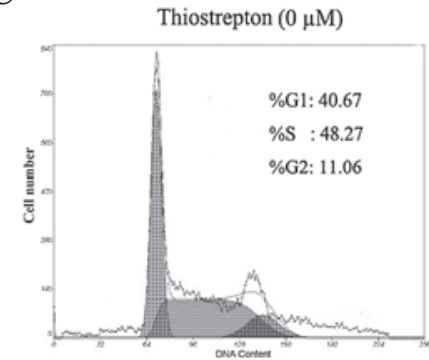

D

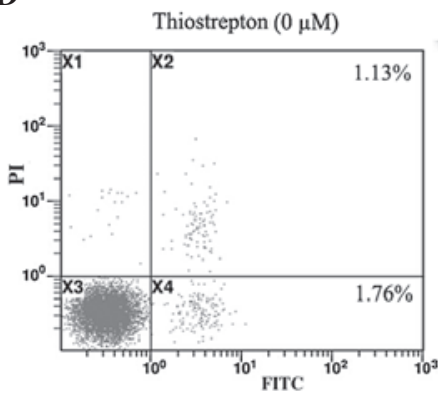

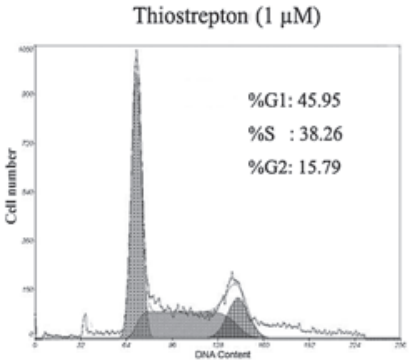

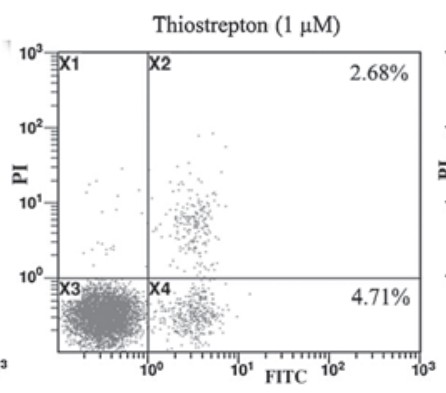

B

Thiostrepton $(\mu \mathrm{M})$

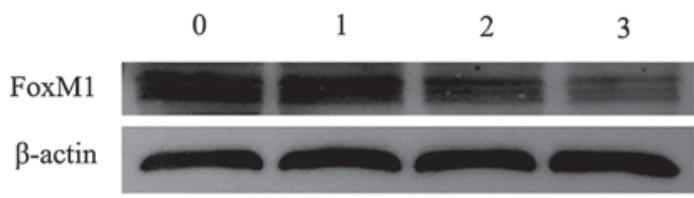

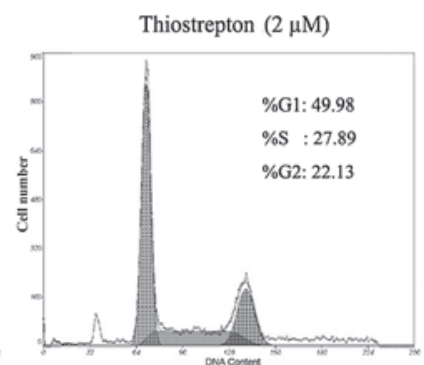
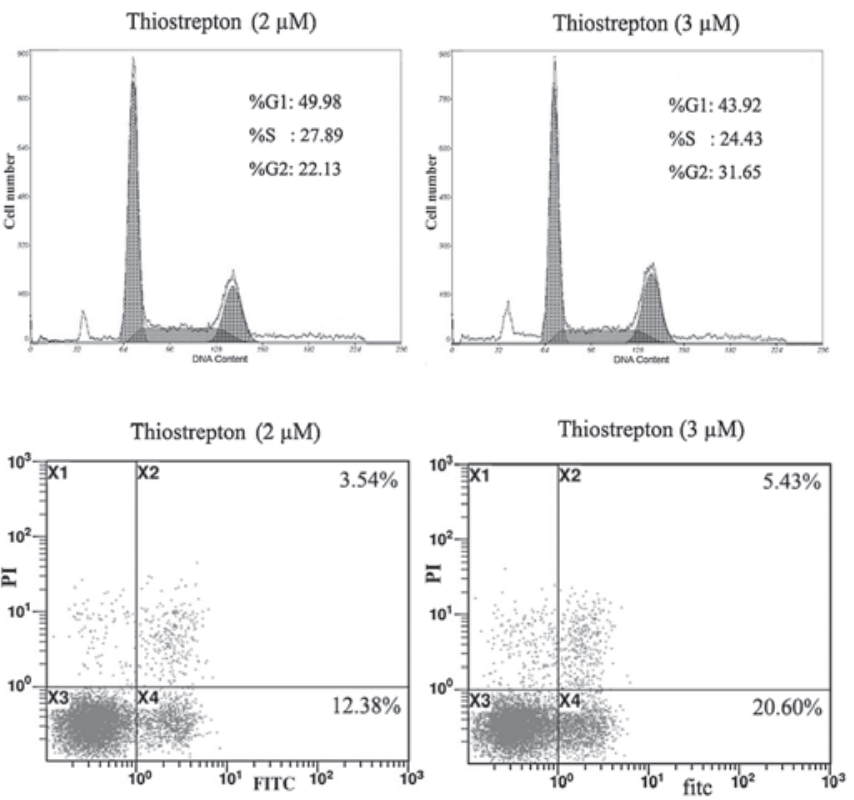

Figure 2. Effect of thiostrepton on the gene and protein expression levels of FoxM1, the cell cycle and apoptosis of Jurkat cells. The Jurkat cells were treated with various concentrations thiostrepton $(0,1,2$ or $3 \mu \mathrm{M})$ for $24 \mathrm{~h}$. (A) Relative mRNA levels of FoxM1 were assessed by reverse transcription-quantitative polymerase chain reaction. The results were calculated using the $2^{-\Delta \Lambda \mathrm{Ct}}$ method. (B) Protein expression levels of FoxM1 were determined using western blot analysis. (C) Cell cycle was examined by flow cytometery. (D) Quantitative analysis of the apoptotic rate. Thiostrepton inhibited the mRNA and protein expression levels of FoxM1, and induced G2/M arrest and apoptosis in a dose-dependent manner. Data are expressed as the mean \pm standard deviation. ${ }^{*} \mathrm{P}<0.05$ vs. other groups. PI, propidium iodide; FITC, fluorescein isothiocyanate.

Flow cytometric analysis of intracellular doxorubicin accumulation. The intracellular accumulation of doxorubicin was measured using a standard fluorescent method (26). The Jurkat cells were cultured in RPMI-1640 containing 10 FBS, with increasing concentrations of thiostrepton $(0,1,2$ or $3 \mu \mathrm{M})$ for $24 \mathrm{~h}$ at $37^{\circ} \mathrm{C}$. The medium was then replaced with RPMI-1640 containing 10\% FBS and doxorubicin $(0.2 \mu \mathrm{M})$, in which the cells were incubated for $1 \mathrm{~h}$ at $37^{\circ} \mathrm{C}$. The cells were then harvested and washed twice in ice-cold PBS. The cell-associated mean fluorescence intensity (MFI) of intracellular doxorubicin was determined using the FACS FC500 flow cytometer with excitation and emission wavelengths of 485 and $585 \mathrm{~nm}$, respectively.

Statistical analysis. Statistical analyses were performed using SPSS 19.0 software (IBM SPSS, Armonk, NY, USA). Data are expressed as the mean \pm standard deviation. The significance of differences between groups were determined using Student's $t$-test or analysis of variance. $\mathrm{P}<0.05$ was considered to indicate a statistically significant difference. The synergistic or antagonistic effects of the two drugs were evaluated, according to the formula $[\mathrm{Q}=\mathrm{Ea}+\mathrm{b} /(\mathrm{Ea}+\mathrm{Eb}-\mathrm{EaxEb})]$. In this equation, $\mathrm{Ea}$

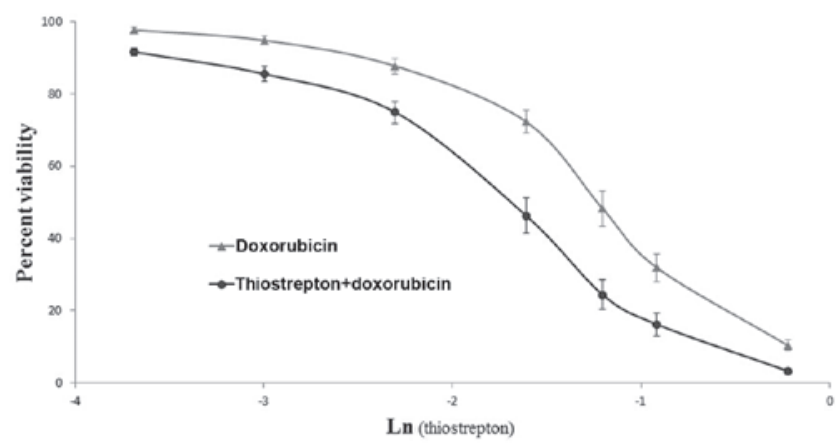

Figure 3. Synergistic effect of thiostrepton and doxorubicin on the viability of the Jurkat cells. The Jurkat cells were co-treated with thiostrepton $(1 \mu \mathrm{M})$ and doxorubicin for $24 \mathrm{~h}$, resulting in a significant decrease in the half maximal inhibitory concentration of doxorubicin between 0.295 and $0.198 \mu \mathrm{mol} / 1$. Data are presented as the percentages relative to the untreated control, as the means \pm standard deviation.

and $\mathrm{Eb}$ represent the individual inhibitory rates of drugs $\mathrm{A}$ and $\mathrm{B}$, respectively, and $\mathrm{Ea}+\mathrm{b}$ represents the inhibitory rate of the combined drug therapy on tumor cell proliferation. Q-values ranging between 0.85 and 1.15 indicated that the effects of 
A
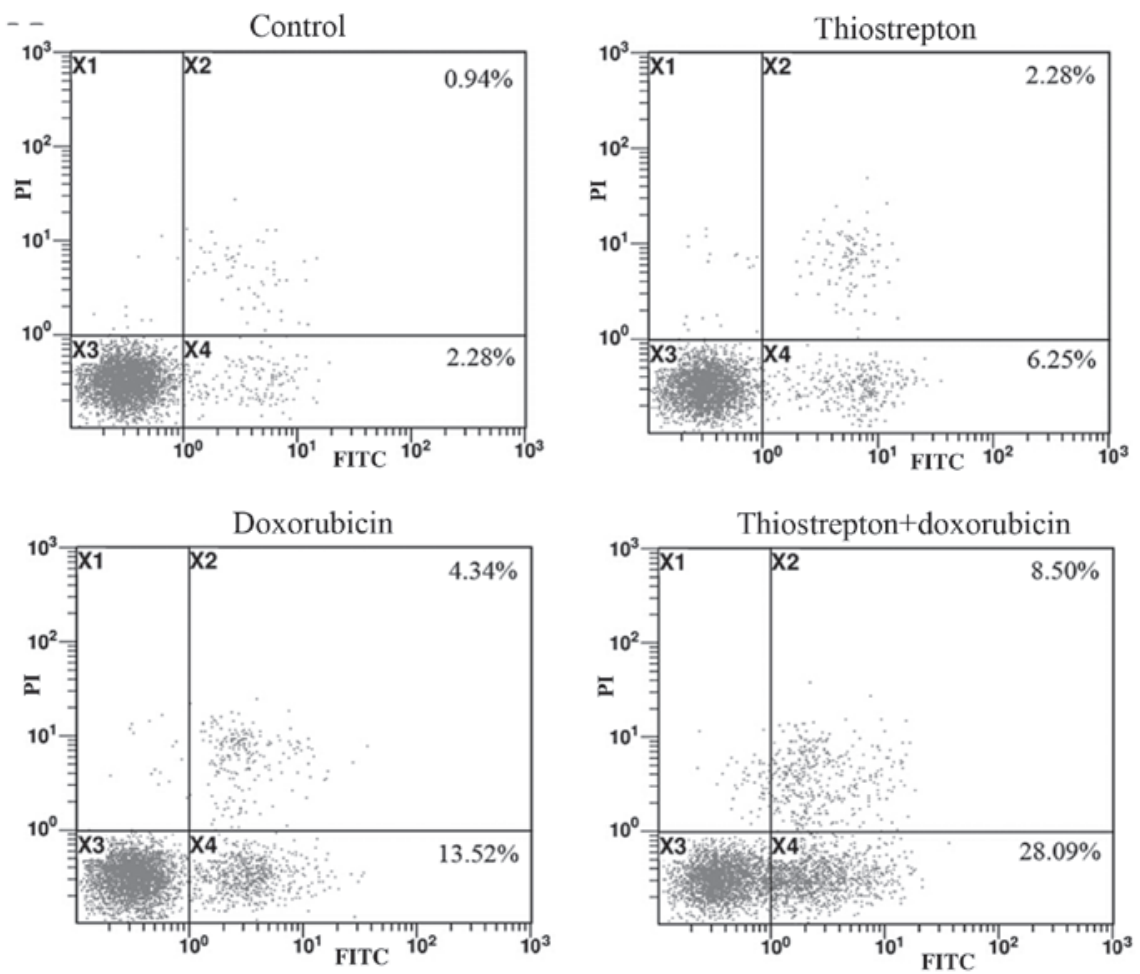

B

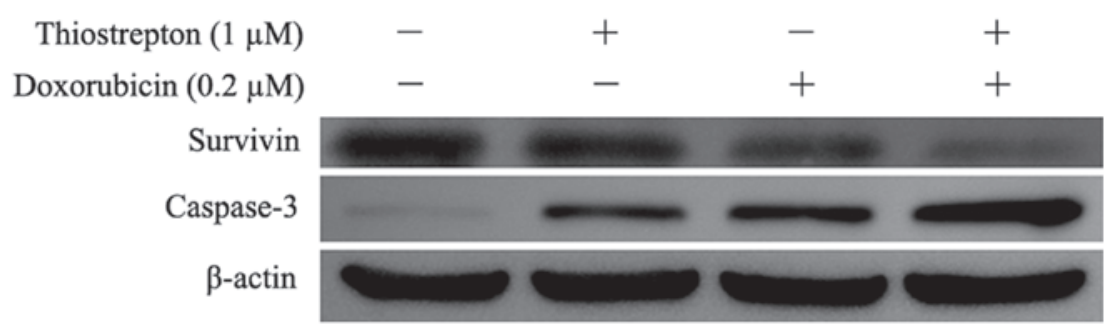

Figure 4. Effect of thiostrepton on doxorubicin-induced apoptosis of Jurkat cells. The Jurkat cells were treated with either thiostrepton (1 $\mu \mathrm{m} / 1)$ or doxorubicin $(0.2 \mu \mathrm{m} / \mathrm{l})$, or with the two drugs combined for $24 \mathrm{~h}$. (A) Treatment with a combination of $1 \mu \mathrm{M}$ thiostrepton and $0.2 \mu \mathrm{M}$ doxorubicin significantly increased Jurkat cell apoptosis compared with treatment with either drug alone $(\mathrm{P}<0.05)$. (B) Expression levels of apoptosis-associated proteins were detected by western blot analysis. PI, propidium iodide; FITC, fluorescein isothiocyanate.

the two drugs were simply additive, whereas Q-values $>1.15$ indicated a synergistic effect, and Q-values $<0.85$ indicated an antagonistic effect for the combined drug therapy.

\section{Results}

Thiostrepton suppresses the viability of Jurkat cells. The Jurkat cells were treated with various concentrations of thiostrepton (0-4 $\mu \mathrm{M})$ for 24,48 or $72 \mathrm{~h}$, and the effect of thiostrepton on cell viability was evaluated using a CCK-8 assay. The results demonstrated that the Jurkat cells were sensitive to thiostrepton, and that thiostrepton inhibited cell growth in a time- and dose-dependent manner $(\mathrm{P}<0.05$; Fig. 1$)$. The $\mathrm{IC}_{50}$ values for thiostrepton in the Jurkat cells at 24,48 and $72 \mathrm{~h}$ were $2.03,1.52$ and $1.29 \mu \mathrm{M}$, respectively.

Thiostrepton-mediated inhibition of FoxM1 induces cell cycle arrest at the G2/M phase and leads to apoptosis of Jurkat cells. To further characterize the mechanism underlying the antileukemia activity of thiostrepton on the Jurkat cells, the
mRNA and protein expression levels of FoxM1 were determined following treatment with various concentrations of thiostrepton $(0,1,2$ or $3 \mu \mathrm{M})$ for $24 \mathrm{~h}$, to examine whether thiostrepton affected cell cycle distribution or apoptosis. The results revealed that thiostrepton inhibited the expression of FoxM1 in a dose-dependent manner, and induced gradual dose-dependent G2/M arrest and apoptosis in the Jurkat cells (Fig. 2).

Inhibition of FoxM1 by thiostrepton enhances the antiproliferative effects of doxorubicin on Jurkat cells. Based on the aforementioned findings, the present study subsequently investigated whether the Jurkat cells with reduced expression of FoxM1 were more sensitive to doxorubicin, a common antileukemic drug used in the treatment of ALL. As shown in Fig. 1, treatment with $1 \mu \mathrm{M}$ thiostrepton for $24 \mathrm{~h}$ resulted in a $7.70 \pm 0.95 \%$ reduction of viable cells $(\mathrm{P}<0.05)$ compared with the control group, therefore, the concentration of $1 \mu \mathrm{M}$ was considered to be a non-cytotoxic dose. The Jurkat cells were incubated with various concentrations of doxorubicin 
A
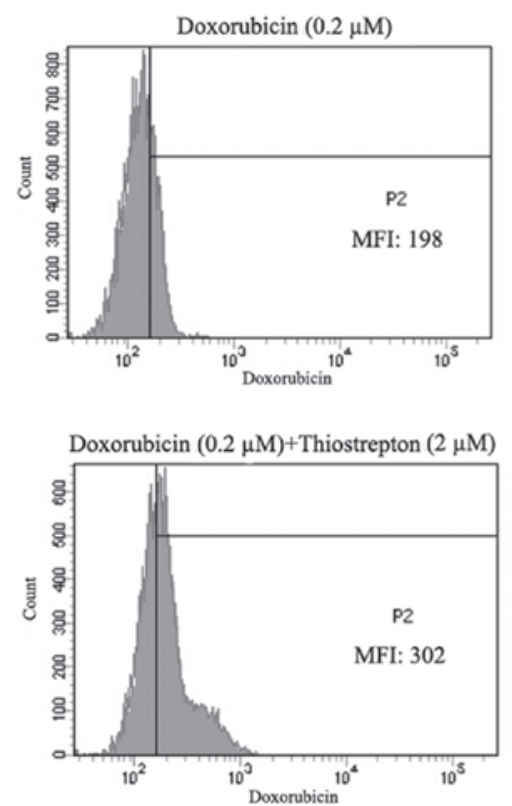
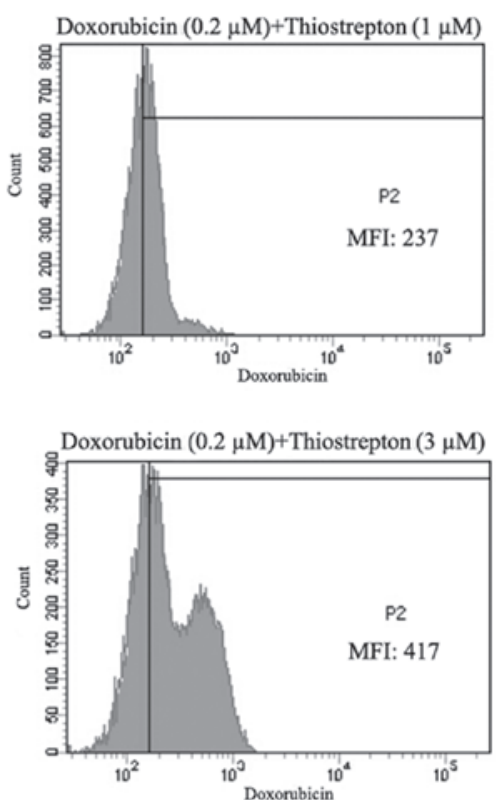

B

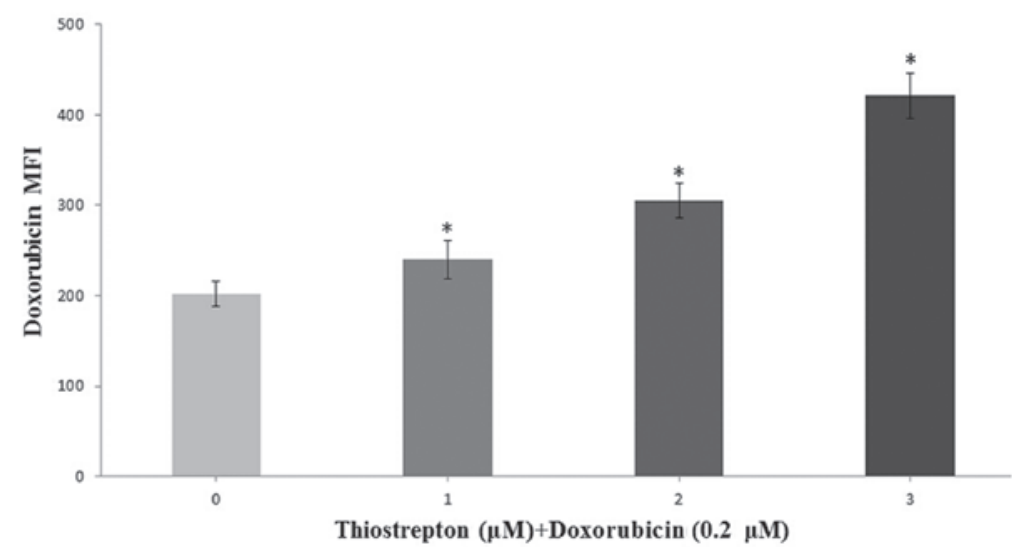

Figure 5. Effect of thiostrepton on intracellular doxorubicin accumulation in Jurkat cells. The Jurkat cells were cultured in various concentrations of thiostrepton $(0,1,2$ or $3 \mu \mathrm{M})$ for $24 \mathrm{~h}$. The thiostrepton was then replaced with doxorubicin $(0.2 \mu \mathrm{M})$ and the cells were incubated for $1 \mathrm{~h}$. The doxorubicin-associated MFI was measured by (A) flow cytometty, with excitation/emission wavelengths of 485/585 nm. (B) Thiostrepton significantly increased doxorubicin-associated MFI in the Jurkat cells, in a dose-dependent manner. Data are expressed as the mean \pm standard deviation. ${ }^{*} \mathrm{P}<0.05$, vs. untreated control. MFI, mean fluorescence intensity.

$(0-0.8 \mu \mathrm{m} / \mathrm{l})$, with or without thiostrepton $(1 \mu \mathrm{m} / \mathrm{l})$, for $24 \mathrm{~h}$. Cell proliferation was measured by performing CCK-8 assays. The results demonstrated that the combined treatment reduced the $\mathrm{IC}_{50}$ value of doxorubicin in the Jurkat cells between 0.295 and $0.198 \mu \mathrm{mol} / 1$ (Fig. 3). Thiostrepton and doxorubicin were identified to synergistically inhibit cell growth, with $Q$ values often $>1.15$ (Table II). It was suggested that the inhibition of FoxM1 by thiostrepton increased the chemosensitivity of Jurkat cells to doxorubicin.

Thiostrepton enhances doxorubicin-induced caspase-mediated apoptosis in Jurkat cells. The present study then examined whether thiostrepton enhances doxorubicin-induced cell apoptosis. The Jurkat cells were treated with a low concentration of either thiostrepton $(1 \mu \mathrm{m} / \mathrm{l})$ or doxorubicin $(0.2 \mu \mathrm{m} / \mathrm{l})$, or treated with the two drugs in combination for $24 \mathrm{~h}$. Flow cytometric analysis revealed that thiostrepton increased doxorubicin-induced apoptosis between 16.98 2 2.87 and
$36.07 \pm 4.18 \%$ ( $\mathrm{P}<0.05$; Fig. 4A). To determine the mechanism leading to the cell apoptosis, the protein expression levels of survivin and caspase-3 were examined. As shown in Fig. 4B, decreases in the expression of survivin were significantly greater in the cells treated with thiostrepton and doxorubicin in combination, compared with those in the single treatment groups. In addition, combination treatment resulted in greater increases in the protein expression of caspase- 3 compared with either drug alone (Fig. 4B).

Thiostrepton increases the intracellular accumulation of doxorubicin in Jurkat cells. The capability of thiostrepton to promote doxorubicin accumulation within Jurkat cells was observed (Fig. 5). The doxorubicin-associated MFI was significantly increased in Jurkat cells, which were treated with various concentrations of thiostrepton $(1,2$ or $3 \mu \mathrm{M})$ for $24 \mathrm{~h}$, which explained the enhanced effects of thiostrepton on doxorubicin cytotoxicity in the Jurkat cells. In addition, thiostrepton 
was observed to elevate the concentration of doxorubicin in the Jurkat cells in a dose-dependent manner $(\mathrm{P}<0.05)$.

Inhibition of FoxM1 by thiostrepton decreases the gene and protein expression levels of GSTpi within the Jurkat cells. To better understand the mechanism by which thiostrepton functionally elevated drug accumulation within the Jurkat cells, the mRNA and protein expression levels of GSTpi were quantitatively measured. The Jurkat cells were treated with various concentrations of thiostrepton $(0,1,2$ or $3 \mu \mathrm{M})$ for $24 \mathrm{~h}$. The gene and protein expression levels of GSTpi in the Jurkat cells decreased significantly when the cells were treated with thiostrepton $(\mathrm{P}<0.05$; Fig. 6). The results indicated that the inhibition of FoxM1 by thiostrepton downregulated the expression of GSTpi in a dose-dependent manner in the Jurkat cells $(\mathrm{P}<0.05)$.

\section{Discussion}

T-ALL is a genetically heterogeneous type of cancer, with $20 \%$ of childhood patients and the majority of adult patients succumbing to mortality from disease resistance or relapse (27). Current standard therapies have unsatisfactory effects on survival rates, and therapy-associated side-effects have led to a focus on identifying novel therapeutic approaches for T-ALL. The present study was the first, to the best of our knowledge, to demonstrate thiostrepton-induced inhibition of FoxM1 in human T-ALL Jurkat cells. The results revealed that thiostrepton inhibited Jurkat cell proliferation and induced apoptosis in a dose-dependent manner. It was also demonstrated that thiostrepton enhanced the chemosensitivity of the Jurkat cells to doxorubicin by enhancing doxorubicin-induced apoptosis and increasing the intracellular accumulation of doxorubicin.

The present study investigated the antileukemic effects of thiostrepton on Jurkat cell proliferation. Thiostrepton significantly inhibited the proliferation of Jurkat cells, and the $\mathrm{IC}_{50}$ value of thiostrepton in the Jurkat cells following $48 \mathrm{~h}$ treatment was $1.52 \mu \mathrm{mol} / \mathrm{l}$, suggesting the sensitivity of the Jurkat cells to thiostrepton was comparable to that of other types of cancer cell $(28,29)$. Furthermore, the results of the present study indicated that thiostrepton induced G2/M cell cycle phase arrest and increased apoptosis in the Jurkat cells. These results demonstrated the efficient antileukemic activity of thiostrepton on Jurkat cells, and suggested that the effects of thiostrepton may depend on the downregulation of FoxM1 target genes, which have been found to be involved in the regulation of cell growth, apoptosis, cell cycle and progression (30).

Previous studies have suggested that silencing FoxM1 enhances sensitivity to chemotherapeutic agents in various types of cancer cells $(14,29)$, therefore, the present study investigated whether the thiostrepton-mediated inhibition of FoxM1 sensitized the Jurkat cells to the effects of chemotherapy. the $\mathrm{IC}_{50}$ value of doxorubicin in the Jurkat cells decreased between 0.295 and $0.198 \mu \mathrm{mol} / 1$ when thiostrepton $(1 \mu \mathrm{mol} / \mathrm{l})$ was used in combination. These results demonstrated that thiostrepton enhanced the doxorubicin cytotoxicity effect within the Jurkat cells through a synergistic effect of thiostrepton and doxorubicin. Therefore, a combination therapy approach using doxorubicin and thiostrepton can improve the effect of

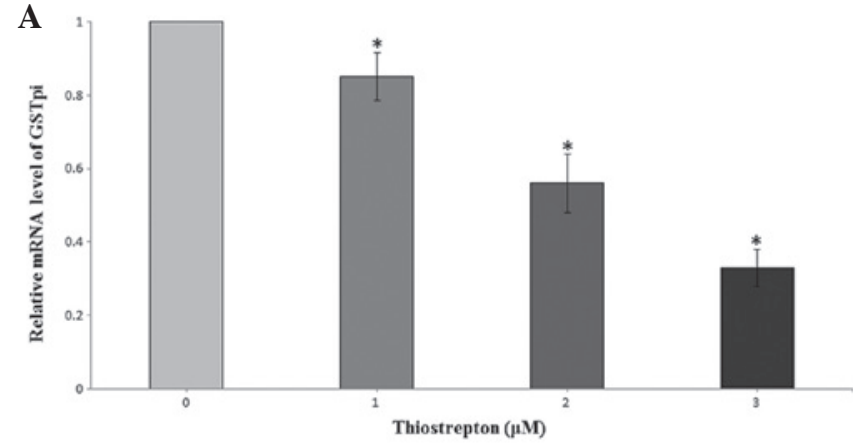

B

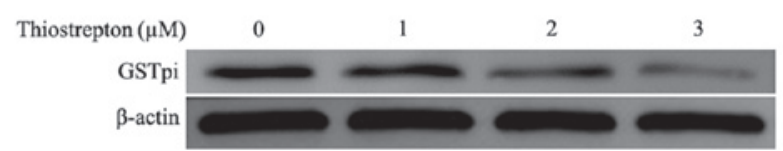

Figure 6. Effects of thiostrepton on the gene and protein expression levels of GSTpi in Jurkat cells. The Jurkat cells were treated with various concentrations of thiostrepton $(0,1,2$ or $3 \mu \mathrm{M})$ for $24 \mathrm{~h}$. (A) Relative mRNA levels of GSTpi were assessed by reverse transcription-quantitative polymerase chain reaction. The results were calculated using the $2^{-\Delta \Delta \mathrm{Ct}}$ method. (B) Protein expression levels of GSTpi were detected by western blot analysis. Thiostrepton inhibited the mRNA and protein expression levels of GSTpi in a dose-dependent manner. Data are expressed as the mean \pm standard decviation. ${ }^{*} \mathrm{P}<0.05$, vs untreated control. GSTpi, glutathione S-transferase pi.

doxorubicin and also reduce the adverse side-effects of doxorubicin, by allowing it to be used at lower doses in treating human T-ALL.

The ability to induce leukemic cell death is critical for successful treatment with chemotherapeutic drugs. In the present study, flow cytometric analysis revealed a more marked increase in apoptosis in cells treated with a combination of thiostrepton and doxorubicin, compared with those treated with either drug alone. To further examine the mechanism underlying the increased efficacy of combination treatment, proteins, which are known to regulate apoptosis, were examined. Survivin is known for its role in preventing apoptosis and conferring resistance to chemotherapeutic agents in various cell lines (31). Specifically, its anti-apoptotic function appears to be associated with the ability to directly or indirectly inhibit caspases (31). In the present study, the cells treated with thiostrepton and doxorubicin combined exhibited a greater decrease in the protein expression of survivin compared with the cells treatedwith doxorubicin only, and thiostrepton also enhanced doxorubicin-induced increases in the protein expression of caspase-3. These results suggested that the inhibition of FoxM1 by thiostrepton sensitized the Jurkat cells to doxorubicin, partly through enhanced apoptosis, possibly via a caspase-3-dependent pathway.

In the present study, thiostrepton significantly elevated the concentration of doxorubicin in Jurkat cells in a dose-dependent manner, while promoting doxorubicin accumulation within the Jurkat cells. To determine the mechanism by which thiostrepton functionally elevated drug accumulation within the Jurkat cells, the expression of GSTpi was measured. Previous studies have demonstrated that the mRNA and protein expression levels of GSTpi are high in Jurkat cells (32). GSTpi is a cytosolic isoform of glutathione s-transferases (GSTs) and its overexpression is associated with resistance to chemotherapy in various types of 
human cell (33). GSTs belong to a large family of functional enzymes, which catalyze the S-conjugation of glutathione (GSH) with a wide variety of electrophilic compounds (34). In addition to increasing GST enzyme activity, alteration of cellular GSH levels has also been observed to contribute the resistance of cells to doxorubicin (35). The results of the present study further revealed that the mRNA and protein expression levels of the GSTpi gene were significantly inhibited by thiostrepton. These results indicated that thiostrepton elevated the sensitivity of Jurkat cells to doxorubicin, partly by increasing the accumulation of intracellular doxorubicin, possibly via downregulating the expression of GSTpi.

In conclusion, the results of the present study demonstrated that thiostrepton significantly inhibited the proliferation, and induced the apoptosis of Jurkat cells. The inhibition of FoxM1 by thiostrepton also chemosensitized the Jurkat cells to doxorubicin by enhancing doxorubicin-induced apoptosis and elevating the accumulation of intracellular doxorubicin. These results suggested that the inhibition of FoxM1 by thiostrepton have wide therapeutic and/or adjuvant applications for T-ALL chemotherapy. Thiostrepton may be particularly useful in young children with resistant or relapsed T-ALL, who currently require more aggressive chemotherapeutic agents.

\section{Acknowledgements}

This study was supported by the NCET-10-0919, 'Taishan Scholar' position, National Natural Science Foundation (nos. 31371321 and 81200601), the Shandong Science and Technology Committee (no. 2010GSF10264), the Foundation of Shandong Educational Committee (nos. J10LC60 and J11LC01) and the Binzhou Science and Technology Committee of China (no. 2011ZC0905).

\section{References}

1. Inaba H, Greaves M and Mullighan CG: Acute lymphoblastic leukaemia. Lancet 381: 1943-1955, 2013.

2. Bhojwani D and Pui CH: Relapsed childhood acute lymphoblastic leukaemia. Lancet Oncol 14: e205-217, 2013.

3. Van Vlierberghe P and Ferrando A: The molecular basis of T cell acute lymphoblastic leukemia. J Clin Invest 122: 3398-3406, 2012.

4. Martelli AM, Lonetti A, Buontempo F, et al: Targeting signaling pathways in T-cell acute lymphoblastic leukemia initiating cells. Adv Biol Regul 56: 6-21, Apr 30, 2014 (Epub ahead of print).

5. Wierstra I and Alves J: FOXM1, a typical proliferation-associated transcription factor. Biol Chem 388: 1257-1274, 2007.

6. Koo CY, Muir KW and Lam EW: FOXM1: From cancer initiation to progression and treatment. Biochim Biophys Acta 1819: 28-37, 2012.

7. Priller M, Pöschl J, Abrão L, et al: Expression of FoxM1 is required for the proliferation of medulloblastoma cells and indicates worse survival of patients. Clin Cancer Res 17 6791-6801, 2011

8. Xue YJ, Xiao RH, Long DZ, et al: Overexpression of FoxM1 is associated with tumor progression in patients with clear cell renal cell carcinoma. J Transl Med 10: 200, 2012.

9. Xia JT, Wang H, Liang LJ, et al: Overexpression of FOXM1 is associated with poor prognosis and clinicopathologic stage of pancreatic ductal adenocarcinoma. Pancreas 41: 629-635, 2012.

10. Xu N, Jia D, Chen W, et al: FoxM1 is associated with poor prognosis of non-small cell lung cancer patients through promoting tumor metastasis. PLoS One 8: e59412, 2013.

11. Li X, Qiu W, Liu B, et al: Forkhead box transcription factor 1 expression in gastric cancer: FOXM1 is a poor prognostic factor and mediates resistance to docetaxel. J Transl Med 11: 204, 2013.
12. Liu LL, Zhang DH, Mao X, Zhang $\mathrm{XH}$ and Zhang B: Over-expression of FoxM1 is associated with adverse prognosis and FLT3-ITD in acute myeloid leukemia. Biochem Biophys Res Commun 446: 280-285, 2014.

13. Myatt SS and Lam EW: The emerging roles of forkhead box (Fox) proteins in cancer. Nat Rev Cancer 7: 847-859, 2007.

14. Halasi M and Gartel AL: Suppression of FOXM1 sensitizes human cancer cells to cell death induced by DNA-damage. PLoS One 7: e31761, 2012.

15. Nakamura S, Hirano I, Okinaka K, et al: The FOXM1 transcriptional factor promotes the proliferation of leukemia cells through modulation of cell cycle progression in acute myeloid leukemia. Carcinogenesis 31: 2012-2021, 2010.

16. Zhang X, Zeng J, Zhou M, et al: The tumor suppressive role of miRNA-370 by targeting FoxM1 in acute myeloid leukemia. Mol Cancer 11: 56, 2012.

17. Mencalha AL, Binato R, Ferreira GM, Du Rocher B and Abdelhay E: Forkhead box M1 (FoxM1) gene is a new STAT3 transcriptional factor target and is essential for proliferation, survival and DNA repair of K562 cell line. PloS one 7: e48160, 2012.

18. Bhat UG, Halasi M and Gartel AL: FoxM1 is a general target for proteasome inhibitors. PLoS One 4: e6593, 2009.

19. Hegde NS, Sanders DA, Rodriguez R and Balasubramanian S: The transcription factor FOXM1 is a cellular target of the natural product thiostrepton. Nat Chem 3: 725-731, 2011.

20. Hedge NS, Sanders DA, Rodriguez R and Balasubramanian S: The transcription factor FOXM1 is a cellular target of the natural product thiostrepton. Nat Chem 3: 725-731, 2011.

21. Gartel AL: Thiostrepton, proteasome inhibitors and FOXM1. Cell Cycle 10: 4341-4342, 2011.

22. Halasi M, Schraufnagel DP and Gartel AL: Wild-type p53 protects normal cells against apoptosis induced by thiostrepton. Cell Cycle 8: 2850-2851, 2009.

23. Ahmed M, Uddin S, Hussain AR, et al: FoxM1 and its association with matrix metalloproteinases (MMP) signaling pathway in papillary thyroid carcinoma. J Clin Endocrinol Metab 97: E1-13, 2012.

24. Wang M and Gartel AL: Micelle-encapsulated thiostrepton as an effective nanomedicine for inhibiting tumor growth and for suppressing FOXM1 in human xenografts. Mol Cancer Ther 10: 2287-2297, 2011.

25. Liu X, Li X, Wang L, et al: Realgar induces apoptosis in the chronic lymphocytic leukemia cell line MEC1. Mol Med Rep 8: 1866-1870, 2013.

26. Ji BS, He L and Liu GQ: Reversal of p-glycoprotein-mediated multidrug resistance by CJX1, an amlodipine derivative, in doxorubicin-resistant human myelogenous leukemia (K562/DOX) cells. Life Sci 77: 2221-2232, 2005.

27. Van Vlierberghe P, Ambesi-Impiombato A, De Keersmaecker K, et al: Prognostic relevance of integrated genetic profiling in adult T-cell acute lymphoblastic leukemia. Blood 122: 74-82, 2013.

28. Bhat UG, Halasi M and Gartel AL: Thiazole antibiotics target FoxM1 and induce apoptosis in human cancer cells. PLoS One 4: e5592, 2009.

29. Lin J, Zheng Y, Chen K, Huang Z, Wu X and Zhang N: Inhibition of FOXM1 by thiostrepton sensitizes medulloblastoma to the effects of chemotherapy. Oncol Rep 30: 1739-1744, 2013.

30. Halasi M and Gartel AL: FOX(M1) news - it is cancer. Mol Cancer Ther 12: 245-254, 2013.

31. Zaffaroni N, Pennati M and Daidone MG: Survivin as a target for new anticancer interventions. J Cell Mol Med 9: 360-372, 2005.

32. Zhou L, Jing Y, Styblo M, Chen Z and Waxman S: Glutathione-S-transferase pi inhibits As2O3-induced apoptosis in lymphoma cells: involvement of hydrogen peroxide catabolism. Blood 105: 1198-1203, 2005.

33. Lourenco GJ, Lorand-Metze I, Delamain MT, et al: Polymorphisms of glutathione S-transferase mu 1, theta 1 and pi 1 genes and prognosis in Hodgkin lymphoma. Leuk Lymphoma 51: 2215-2221, 2010.

34. Ballerini S, Bellincampi L, Bernardini S, Iori R, Cortese C and Federici G: Analysis of GSTP1-1 polymorphism using real-time polymerase chain reaction. Clin Chim Acta 329: $127-132,2003$.

35. Song YN, Guo XL, Zheng BB, et al: Ligustrazine derivate DLJ14 reduces multidrug resistance of K562/A02 cells by modulating GST $\pi$ activity. Toxicol In Vitro 25: 937-943, 2011. 